\title{
The effect of shoe type and fatigue on strike index and spatiotemporal parameters of running
}

Citation for published version (APA):

Mann, R., Malisoux, L., Urhausen, A., Statham, A., Meijer, K., \& Theisen, D. (2015). The effect of shoe type and fatigue on strike index and spatiotemporal parameters of running. Gait \& Posture, 42(1), 91-95. https://doi.org/10.1016/j.gaitpost.2015.04.013

Document status and date:

Published: 01/01/2015

DOI:

10.1016/j.gaitpost.2015.04.013

Document Version:

Publisher's PDF, also known as Version of record

Document license:

Taverne

Please check the document version of this publication:

- A submitted manuscript is the version of the article upon submission and before peer-review. There can be important differences between the submitted version and the official published version of record.

People interested in the research are advised to contact the author for the final version of the publication, or visit the DOI to the publisher's website.

- The final author version and the galley proof are versions of the publication after peer review.

- The final published version features the final layout of the paper including the volume, issue and page numbers.

Link to publication

\footnotetext{
General rights rights.

- You may freely distribute the URL identifying the publication in the public portal. please follow below link for the End User Agreement:

www.umlib.nl/taverne-license

Take down policy

If you believe that this document breaches copyright please contact us at:

repository@maastrichtuniversity.nl

providing details and we will investigate your claim.
}

Copyright and moral rights for the publications made accessible in the public portal are retained by the authors and/or other copyright owners and it is a condition of accessing publications that users recognise and abide by the legal requirements associated with these

- Users may download and print one copy of any publication from the public portal for the purpose of private study or research.

- You may not further distribute the material or use it for any profit-making activity or commercial gain

If the publication is distributed under the terms of Article $25 \mathrm{fa}$ of the Dutch Copyright Act, indicated by the "Taverne" license above, 


\title{
The effect of shoe type and fatigue on strike index and spatiotemporal parameters of running
}

\author{
Robert Mann a,b, Laurent Malisoux a , Axel Urhausen ${ }^{\mathrm{a}, \mathrm{c}}$, Andrew Statham ${ }^{\mathrm{d}}$, \\ Kenneth Meijer $^{\mathrm{b}}$, Daniel Theisen ${ }^{\mathrm{a}, *}$ \\ ${ }^{a}$ Sports Medicine Research Laboratory, Luxembourg Institute of Health, 76, rue d'Eich, L-1460 Luxembourg, Luxembourg \\ ${ }^{\mathrm{b}}$ NUTRIM School for Nutrition, Toxicology and Metabolism, Maastricht University, Universiteitssingel 40, 6229 ER, Maastricht, The Netherlands \\ ' Sports Clinic, Centre Hospitalier de Luxembourg, 78, rue d'Eich, L-1460 Luxembourg, Luxembourg \\ ${ }^{\mathrm{d}}$ ATO-gear, 20, Torenallee, 5617 BC, Eindhoven, The Netherlands
}

\section{A R T I C L E I N F O}

\section{Article history:}

Received 10 December 2014

Received in revised form 17 April 2015

Accepted 20 April 2015

\section{Keywords:}

Pressure insole

Running style

Foot strike pattern

Detrended fluctuation analysis

\begin{abstract}
A B S T R A C T
We aimed to observe differences in running style parameters and the stride-to-stride coefficient of variation and correlative patterns using detrended fluctuation analysis (DFA) between conventional and first-time minimalistic shoe use. We also aimed to study the effect of fatigue on these parameters. 26 recreational runners were tested using a pressure insole device on a treadmill whilst wearing conventional (CONV) and minimalistic (MIN) shoes. They then performed a prolonged running bout simulating a fatiguing training session, before being tested a second time in both shoe types. Average values of strike index (initial ground contact point on the footsole expressed as a percentage of total sole length) were not significantly different between CONV [25.7 $\pm 14.6 \%$ (unfatigued), $23.1 \pm 11.1 \%$ (fatigued)] and MIN [28.9 $\pm 19.1 \%$ (unfatigued), $26.7 \pm 17.6 \%$ (fatigued)] $(p=0.501$ ). The fatigued state also yielded a similar strike index compared to the unfatigued state $(p=0.661)$. An overall trend in decreased inter-stride correlative patterns of strike index was observed in MIN compared to CONV $(p=0.075)$. No differences in contact time, flight time, stride time, duty factor, stride length and stride frequency were found between shoe types. A trend in reduced flight time $(p=0.078)$ and therefore increased duty factor $(p=0.053)$ was observed due to fatigue. We conclude that in recreational runners, no meaningful, acute adaptation in running style occurs as a result of first-time MIN use. Similarly, runners were able to maintain their running style after a prolonged running bout.
\end{abstract}

(c) 2015 Elsevier B.V. All rights reserved.

\section{Introduction}

Shoe type has been thought to affect the running style, and a handful of studies comparing minimalistic (MIN - low profile, flexible sole, reduced or zero drop, wide toe-box and no motion control or heavy cushioning) and conventional (CONV) running shoes have been published [1-4]. Popular belief is that MIN encourage a non-rearfoot strike (non-RFS) running pattern [5], thereby lowering vertical loading rates which have been associated with the occurrence of injuries [6]. However, Willy et al. (2014) observed higher vertical loading rates when MIN were used compared to CONV, and interestingly a more dorsiflexed foot at footstrike [3]. Similarly, a study comparing the ground reaction

\footnotetext{
* Corresponding author. Tel.: +352 26917 824; fax: +352 26970 871; mobile: +352 691226611

E-mail address: daniel.theisen@lih.lu (D. Theisen).
}

forces between racing flats and CONV found that loading rates and peak vertical impact force were significantly higher when male runners used racing flats [7]. A shorter stride length $\left(S_{\text {length }}\right)$ and higher stride frequency ( $S_{\text {frequency }}$ ) have been found with MIN compared to CONV [4]. Squadrone and Gallozzi [1] found a significantly reduced contact time ( $\left.T_{\text {contact }}\right)$ and increased strike index (SI) with MIN. SI is a continuous measure of strike pattern expressing the initial contact point on the foot sole as a percentage of the total sole length, with $0 \%$ at the heel $[8,9]$. In contrast to the above-mentioned studies [3,4,7], Squadrone and Gallozzi [1] tested habitually barefoot runners, a minority among modern runners. Indeed it has been observed that $88-94 \%$ of recreational, shod runners adopt a rearfoot strike (RFS) pattern [10,11]. Therefore a consensus on the effect of shoe type on running style, especially foot strike pattern and temporal parameters, has not yet been reached.

The effect of physical fatigue on running style also remains elusive. Fatigue due to training and extended bouts of running has 
been theorised as a potential mechanism of injury [12-15]. Researchers have looked into identifying biomechanical differences between measurements taken before and after a fatiguing running bout, yielding conflicting results for vertical loading rate $[12,15], S_{\text {length }}[13,16]$ and $S_{\text {frequency }}[16,17]$ for example. Willems et al. [18] concluded that several plantar pressure patterns change as a result of a $20 \mathrm{~km}$ race and could contribute to the development of injuries, whereas Alfuth and Rosenbaum [19] found no differences in plantar pressure measurements before and after a $10 \mathrm{~km}$ run. These conflicting findings could be due to the variations in protocols used to fatigue the runners. The importance of recreating typical training conditions (i.e. duration and intensity) has been highlighted and recommended as these are the conditions during which most injuries occur [20].

Our main aim was to observe any differences in SI and spatiotemporal parameters between first-time MIN, and CONV use. A second aim was to identify any fatigue effect, induced by a prolonged running bout designed to mimic typical running activity, on running style parameters. Finally, we tested the interaction of the shoe type and fatigue and its influence on running style. We hypothesised that running in MIN would increase the SI whereas fatigue would decrease it.

\section{Methods}

\subsection{Participants}

A sample size calculation with a power of $80 \%$, a significance level set at $p=0.05$ and an expected SI mean difference based on minimal detectible change of $4.9 \%$ [9] between shoe conditions yielded a required 20 participants for this study. Runners over the age of 18 , injury-free during the previous 12 months, running a minimum of twice a week on average and with an average session duration of $\geq 45$ min with CONV were included. Runners were contacted via leaflets at races, sport shops and public training locations as well as through direct contact with participants of previous cohort studies from our laboratory. Runners already familiar with MIN, unfamiliar with treadmill running, deemed unfit to undertake strenuous exercise (by way of a standardised cardiovascular screening questionnaire) or requiring orthopaedic insoles for running were excluded. Written informed consent was acquired; the study was approved by the National Research Ethics Committee of Luxembourg (CNER N ${ }^{\circ}$ : 201403/06).

\subsection{Protocol}

Participants declared their preferred running speed (PRS) defined as the speed they could maintain for a typical running session, and average session duration over the last 12 months. They provided details regarding past and current running shoe use. All testing was performed on a treadmill (Woodway, PPS70 Plus, Germany) in two different shoe types: MIN (0 mm drop, $5 \mathrm{~mm}$ overall stack height, $158 \pm 15$ g average shoe mass, very flexible) and CONV (10 mm drop, $26 \mathrm{~mm}$ heel stack height, $284 \pm 25 \mathrm{~g}$ average shoe mass). The order of shoe testing was randomised, and runners were equipped with the Runalyser (TNO, Eindhoven, The Netherlands), a pressure-sensitive insole device which is inserted into the shoe and designed to measure pressure location and temporal parameters [9]. The pre-fatigue acquisition protocol started with a 5 -min warm-up at $85 \%$ of the PRS. Then treadmill speed was increased to the PRS and participants continued running for another $5 \mathrm{~min}$. This resulted in $10 \mathrm{~min}$ of running, enough time to provoke short-term adaptation to shoe type [21]. Pressure data was acquired during the final 2 min at the PRS along with heart rate (HR) and rate of perceived exertion (RPE) using the visual analogue scale from 6 to 20 [22]. After a 5-min recovery period, the procedure was repeated with the other shoe type. After a 2-min break, participants completed a fatiguing running bout using their own, habitual running shoes. Duration was defined as $120 \%$ of a typical running session duration minus the 20 min pre-fatigue acquisition period. If participants felt they could not complete the predefined duration, slight adjustments in speed were made according to the RPE and HR which were recorded every $5 \mathrm{~min}$. We ensured that $\mathrm{RPE}$ remained $<17$ throughout. After completing the fatiguing running bout, the acquisition protocol for both shoe types was repeated (in the same order) with 2-min breaks for the participant to change shoes and install the Runalyser.

\subsection{Data analysis}

Data of both feet were averaged for all acquisitions, and SI, contact time $\left(T_{\text {contact }}\right)$, flight time $\left(T_{\text {flight }}\right)$, stride time $\left(T_{\text {stride }}\right)$, duty factor (DF, $\left.T_{\text {contact }} / T_{\text {stride }} \times 100\right)$, $S_{\text {length }}$ and $S_{\text {frequency }}$ were calculated using custom-made MATLAB (Mathworks Inc., USA) algorithms as previously described [9]. Based on previously published cut-offs [9], runners were subdivided as RFS (SI $<43.9 \%$ ) or nonRFS (SI > 43.9\%) runners using their pre-fatigue, CONV data. Using the full 2-min data acquisitions, coefficient of variation (CV) of each parameter was calculated providing information on the stride variability. Detrended fluctuation analysis (DFA) was performed on all parameters, to detect any presence of stride-to-stride correlative patterns within each time series $[23,24]$. This technique calculates a scaling exponent value known as $\alpha$, which can be interpreted as follows: $\alpha=0.5$ represents white noise or the absence of any correlation, $\alpha>0.5$ signifies that long-range correlations are present (as $\alpha$ increases, so does the strength of the correlation), meaning that a given stride is correlated with one or more previous strides. Finally, an $\alpha<0.5$ signifies the presence of anti-correlations, meaning that a shorter stride is more likely to be followed by a longer one, for example.

\subsection{Statistics}

Linear mixed models with "shoe type" and "fatigue" as fixed effects and "subject" as random slopes and intercepts were used to test all variables measured with the unstructured term applied. The statistical software package SPSS for Windows version 20 was used. Statistical significance was accepted at $p<0.05$.

\section{Results}

27 runners were tested, and data for one participant who was unable to complete the fatiguing running bout due to pain in the lower extremity was discarded. Table 1 provides a description of the 26 runners retained for the analysis. Four runners (15.4\%) were identified as non-RFS runners (no significant differences in demographics were observed between non-RFS and RFS runners). The average running duration between acquisitions of the same shoe type was $73.7 \pm 13.1 \mathrm{~min}$,

Table 1

Descriptives and training characteristics of the 26 participants.

\begin{tabular}{ll}
\hline RFS runners & $n=22(84.6 \%)$ \\
Sex (male) & $14(63.6 \%)$ \\
Age $(\mathrm{y})$ & $40 \pm 8$ \\
Height $(\mathrm{m})$ & $1.75 \pm 0.08$ \\
Weight $(\mathrm{kg})$ & $70 \pm 10$ \\
BMI $\left(\mathrm{kg} / \mathrm{m}^{2}\right)$ & $22.9 \pm 2.0$ \\
Running experience (years) & $9.8 \pm 7.8$ \\
Average number of sessions/week & $3.3 \pm 1.1$ \\
Average session duration $(\mathrm{min})$ & $63.2 \pm 13.2$ \\
Preferred running speed $(\mathrm{km} / \mathrm{h})$ & $10.7 \pm 1.3$ \\
Total mileage last 12 months $(\mathrm{km})$ & $1352 \pm 758$ \\
Months run last 12 months & $11.8 \pm 0.6$
\end{tabular}

RFS: rearfoot strike; BMI: body mass index; values are mean \pm SD. 
and the average $\mathrm{HR}$ at the end of the fatiguing protocol was $164 \pm 15 \mathrm{bpm}$, representing $91 \pm 8 \%$ of the overall maximal HR estimated for each individual as 220 -age. Participants reported a mean final RPE score of $14.0 \pm 1.5$ (intensity = "hard"). Although overall average values of SI were higher for MIN [28.9 $\pm 19.1 \%$ (unfatigued) and $26.7 \pm 17.6 \%$ (fatigued)] than for CONV [25.7 $\pm 14.6 \%$ (unfatigued) and $23.1 \pm 11.1 \%$ (fatigued)], these differences were not significant $(p=0.501)$ (Fig. 1A). The dotted line in Fig. 1A delineates the cut-off value for SI between a RFS and non-RFS running pattern, with data points below the dotted line representing a RFS. No significant differences due to fatigue state were observed $(p=0.661)$. Similarly, no shoe effect $(p=0.374)$ or fatigue effect $(p=0.305)$ was found for the CV of SI (Fig. 1B). DFA of SI displayed an overall trend towards a reduced $\alpha(p=0.075)$ in CONV, which was primarily due to the RFS group ( $p=0.051$ ); no fatigue effect was observed for the $\alpha$ of SI ( $p=0.841$ ) (Fig. 1C).

We observed an overall trend for a higher DF in the fatigued condition ( $p=0.053)$, which was due to the trend present in the RFS group ( $p=0.052)$ (Fig. 2D). Similarly, a trend in reduced $T_{\text {flight }}$ with fatigue was also visible overall $(p=0.078)$ and in particular for the RFS group $(p=0.053)$ (Fig. 2B). None of the other
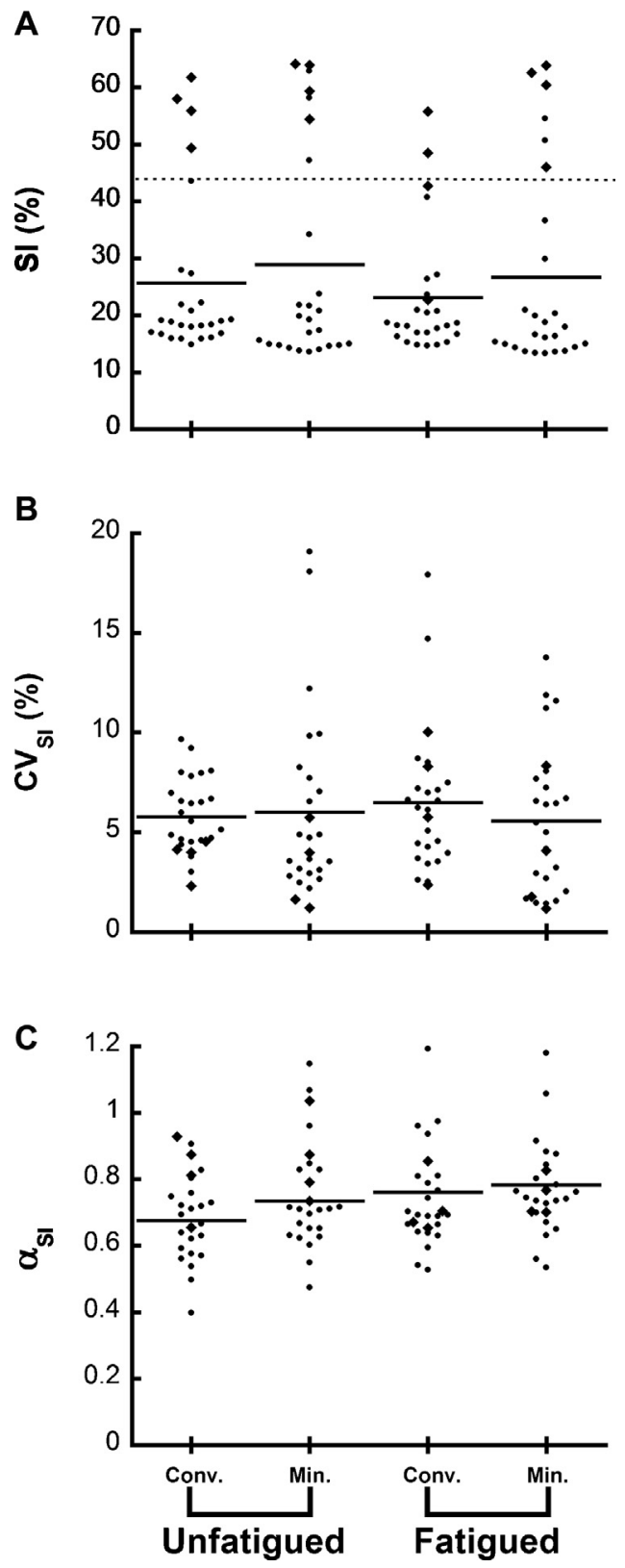

Fig. 1. Dispersion of strike index as measured using the Runalyser (A), the coefficient of variation of strike index (B) and detrended fluctuation analysis of strike index $(C)$ for each of the four running conditions. The dotted line represents the cut-off between a rearfoot and a non-rearfoot strike running pattern. Habitually rearfoot strike runners, $\bullet$ habitually non-rearfoot strike runners based on unfatigued, conventional shoe acquisition. Horizontal lines represent the means. parameters displayed any significant differences or trends for shoe type or fatigue effect (Fig. 2). This was also the case for the CV and the $\alpha$ of all these parameters, as well as for the shoe type*fatigue interactions. The data for all parameters tested in all conditions is available as supplementary data online.

\section{Discussion}

Our main aim was to observe differences in SI and spatiotemporal parameters between running with CONV, and first-time running with MIN. Overall, we did not find any significant differences between shoe types and between the unfatigued and fatigued state. Therefore, except for a tendency towards a reduced $\alpha$ of SI when using CONV, we could not confirm our hypothesis within the conditions of this experiment. Only three habitual RFS runners changed to a non-RFS pattern when running in MIN in the unfatigued condition. The overall increase of $3.4 \%$ SI did not meet the minimal detectible change expected. Despite the MIN in this study having only a $5 \mathrm{~mm}$ stack height, this aspect alone did not incur an overall significant short-term change in running strike pattern. Our results agree with two recent studies also finding no difference in SI between shoe types $[25,26]$. Yet both observed a reduced $T_{\text {contact }}$ for the MIN condition, which was not the case in our study. Similarly, we observed that changing the shoe type was not enough to provoke a significant acute change in $S_{\text {length }}$ and

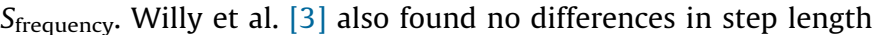
and frequency between MIN and CONV, and neither did Willson et al. [2] for step length and $T_{\text {contact }}$ McCallion et al. [26] did observe a shorter $T_{\text {stride, }}$ a shorter $T_{\text {contact }}$ and a higher $S_{\text {frequency }}$ in the MIN condition in 14 competitive runners at $13.0 \pm 1.0$ and $16.1 \pm 1.3 \mathrm{~km} / \mathrm{h}$. However, they did not observe systematic differences for both speeds, and the clinical relevance of the differences they observed is difficult to determine. Their subjects underwent a 4-min familiarisation trial with all shoe types on a previous occasion, were more competitive, ran faster and most habitually used a midfoot strike running pattern. In all, those runners were perhaps more predisposed to adapt $T_{\text {stride, }} T_{\text {contact }}$ and $S_{\text {frequency }}$ according to shoe type than our runners. $T_{\text {flight }}$ and $T_{\text {stride }}$ were not statistically different between the two shoe types in the present study. Another reason for differences in findings between studies could be related to the varying types of MIN used. There is no consensus on a definition of minimalism, and care must be taken when comparing results of studies using different brands and models of minimalistic shoes [4]. So far the Nike Free 3.0 (17 mm heel stack height, $4 \mathrm{~mm}$ drop) $[3,4]$, Merrell Pace Glove (9.5 $\mathrm{mm}$ overall stack height, $0 \mathrm{~mm}$ drop) [25] or the Vibram 5 Fingers Bikila ( $3 \mathrm{~mm}$ rubber sole, $0 \mathrm{~mm}$ drop) $[1,2,26]$ for example have been tested. Our study used a minimalistic shoe comparable to the latter model.

Stride variability (CV) and stride-to-stride correlative patterns (DFA) of spatiotemporal parameters were also analysed. Other than the observed trend in $\alpha$ of SI, it seems that first-time running in MIN does not have a short-term effect on the variability or longrange correlations of strike pattern and spatiotemporal parameters. A truly lower $\alpha$ of SI would indicate that CONV induces a more random strike pattern; i.e. strides are less correlated with each other than when running in MIN, especially for habitual RFS runners. In such a case, CONV could provide more possibility to change the strike pattern between strides, most likely due to the familiar sensation provided by the cushioning. MIN provided an unfamiliar feel for all of our participants mainly due to the absence of a thick midsole or crash-pad (often commented on by the runners), possibly invoking greater awareness of the foot-ground contact, leading to a more correlated strike pattern. However, this discussion is speculative since only trends were observed. Further analyses of long-range correlative patterns of SI are warranted, along with comparing groups of runners habitually using minimalistic and conventional running shoes. 

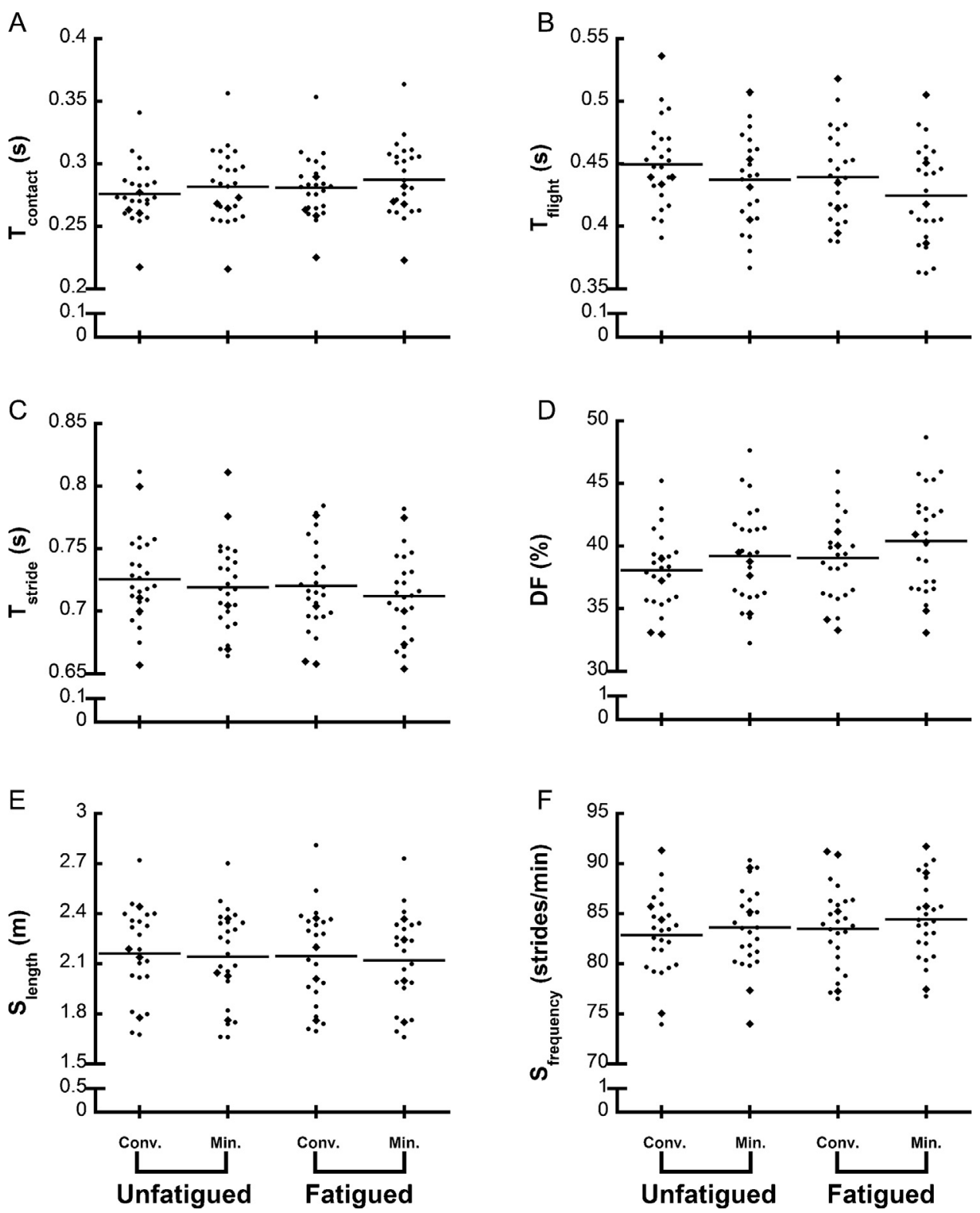

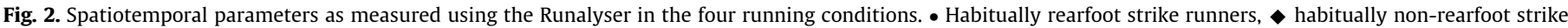
runners based on unfatigued, conventional shoe acquisition. Horizontal lines represent the means.

Our second aim was to observe the effect of fatigue on running style. None of the parameters tested yielded significant changes. With an overall reduction of $2.4 \% \mathrm{SI}$ in the fatigued condition, this does not meet our minimal detectible change of $4.9 \%$ and therefore not a relevant difference. The four non-RFS runners maintained this running style using the MIN in the unfatigued and fatigued condition. Two runners reduced their SI to below the cut-off while using the CONV in the fatigued state. Of the 22 RFS runners, three adopted a non-RFS using MIN in the unfatigued state, and two maintained this style in the fatigued state. No universal adaptation to shoe type was identified therefore, and any change appears to be individual specific. Contrarily, Larson et al. [11] observed increased RFS at the $32 \mathrm{~km}$ mark of a marathon, concluding that muscle fatigue induced a change in running style.

The trend in increased DF was provoked by the trend in decreased $T_{\text {flight }}$ and unchanged $T_{\text {contact }}$ in the fatigued state. A significant reduction in $T_{\text {flight }}$ has been observed 3 hours after a $166 \mathrm{~km}$ ultra-marathon [17]. These authors hypothesised that the reduction in propulsive forces due to fatigue resulted in a 'smoother' run as $S_{\text {frequency }}$ was also significantly increased and vertical oscillation of the spring-mass system significantly reduced. Although our fatiguing protocol does not compare, the same anatomical reasoning could apply to explain this trend. Another study found a prolonged $T_{\text {contact }}$ of $2 \%$ after a $20 \mathrm{~km}$ run and assumed a shortened $T_{\text {flight }}$ [18], however, clinically this finding was deemed of little importance. $T_{\text {contact }}$ in the present study was increased by a comparable $1.8 \%$ as a result of fatigue. Alfuth and Rosenbaum [19] found no change in plantar sensitivity or loading after a $10 \mathrm{~km}$ run simulating moderately exhausting exertion with a max HR of $173 \pm 13 \mathrm{bpm}$ and a mean RPE of $14.5 \pm 2.0$, similar to that of the present study.

A limitation was that we tested our subjects on a treadmill and not in their natural environment. The treadmill used had a good dampening system with rubber slats as the running surface, possibly yielding different results to other running surfaces. Some of our observations are based on trends in the data, and not significant findings. Inclusion of more runners in future studies could shed more light on the relevance of these findings. Although 
$15.4 \%$ non-RFS runners is consistent with the literature [11], no meaningful analyses on this subgroup of four could be conducted. Further, SI provides a reliable measure of the initial contact point on the foot sole with the ground, yet additional loading parameters such as peak vertical impact force and loading rate could provide more accurate information on the interaction of shoe type and loading mechanisms.

\section{Conclusion}

Overall, our findings demonstrate that regular, recreational runners do not display an intuitive and significant adaptation in running style when asked to run using CONV and MIN consecutively. This supports previous work suggesting that MIN do not in themselves provide enough stimuli to provoke any shortterm adaptation in running style compared to running in CONV in first-time users. Further investigation of the trend in increased randomness of the strike pattern using CONV, and how this relates to injury is needed. Physical fatigue induced by a typical training intensity had no significant effect on the SI or spatiotemporal parameters at the preferred running speed, meaning recreational runners are able to maintain their running style throughout a fatiguing training session.

\section{Conflicts of interest statement}

None to declare.

\section{Acknowledgments}

The present study was financially supported by the National Research Fund, Luxembourg (AFR Robert Mann: ref.1102562). Thanks go to Dr Marc Hoppenbrouwers of TNO, Eindhoven, The Netherlands for his technical support, and to the study participants for their valuable contribution.

\section{Appendix A. Supplementary data}

Supplementary data associated with this article can be found, in the online version, at http://dx.doi.org/10.1016/j.gaitpost. 2015.04.013.

\section{References}

[1] Squadrone R, Gallozzi C. Biomechanical and physiological comparison of barefoot and two shod conditions in experienced barefoot runners. J Sports Med Phys Fitness 2009;49(1):6-13.

[2] Willson JD, Bjorhus JS, Williams 3rd DS, Butler RJ, Porcari JP, Kernozek TW. Short-term changes in running mechanics and foot strike pattern after introduction to minimalistic footwear. PM R 2014;6(1):34-43.
[3] Willy RW, Davis IS. Kinematic and kinetic comparison of running in standard and minimalist shoes. Med Sci Sports Exerc 2014;46(2):318-23.

[4] Bonacci J, Saunders PU, Hicks A, Rantalainen T, Vicenzino BG, Spratford W. Running in a minimalist and lightweight shoe is not the same as running barefoot: a biomechanical study. Br J Sports Med 2013;47(6):387-92.

[5] Lieberman DE. What we can learn about running from barefoot running: an evolutionary medical perspective. Exerc Sport Sci Rev 2012;40(2):63-72.

[6] Zadpoor AA, Nikooyan AA. The relationship between lower-extremity stress fractures and the ground reaction force: a systematic review. Clin Biomech (Bristol Avon) 2011;26(1):23-8.

[7] Logan S, Hunter I, JTH JT, Feland JB, Parcell AC. Ground reaction force differences between running shoes, racing flats, and distance spikes in runners. J Sports Sci Med 2010;9(1):147-53.

[8] Cavanagh PR, Lafortune MA. Ground reaction forces in distance running. J Biomech 1980;13(5):397-406.

[9] Mann R, Malisoux L, Brunner R, Gette P Urhausen A Statham A et al. Reliability and validity of pressure and temporal parameters recorded using a pressure-sensitive insole during running. Gait Posture 2014;39(1):455-9.

[10] Kasmer ME, Liu XC, Roberts KG, Valadao JM. Foot-strike pattern and performance in a marathon. Int J Sports Physiol Perform 2013;8(3):286-92.

[11] Larson P, Higgins E, Kaminski J, Decker T, Preble J, Lyons D, et al. Foot strike patterns of recreational and sub-elite runners in a long-distance road race. J Sports Sci 2011;29(15):1665-73.

[12] Christina KA, White SC, Gilchrist LA. Effect of localized muscle fatigue on vertical ground reaction forces and ankle joint motion during running. Hum Mov Sci 2001;20(3):257-76

[13] Clansey AC, Hanlon M, Wallace ES, Lake MJ. Effects of fatigue on running mechanics associated with tibial stress fracture risk. Med Sci Sports Exerc 2012;44(10):1917-23.

[14] Derrick TR, Dereu D, McLean SP. Impacts and kinematic adjustments during an exhaustive run. Med Sci Sports Exerc 2002;34(6):998-1002.

[15] Gerlach KE, White SC, Burton HW, Dorn JM, Leddy JJ, Horvath PJ. Kinetic changes with fatigue and relationship to injury in female runners. Med Sci Sports Exerc 2005;37(4):657-63.

[16] Willson JD, Kernozek TW. Plantar loading and cadence alterations with fatigue. Med Sci Sports Exerc 1999;31(12):1828-33.

[17] Morin JB, Tomazin K, Edouard P, Millet GY. Changes in running mechanics and spring-mass behavior induced by a mountain ultra-marathon race. J Biomech 2011;44(6):1104-7.

[18] Willems TM, De Ridder R, Roosen P. The effect of a long-distance run on plantar pressure distribution during running. Gait Posture 2012;35(3): 405-9.

[19] Alfuth M, Rosenbaum D. Long distance running and acute effects on plantar foot sensitivity and plantar foot loading. Neurosci Lett 2011:503(1):58-62.

[20] Dierks TA, Davis IS, Hamill J. The effects of running in an exerted state on lower extremity kinematics and joint timing. J Biomech 2010;43(15): 2993-8.

[21] Delattre N, Chambon N, Berton E, Gueguen N, Rao G. Effect of time during a running session with minimal footwear. Comput Methods Biomech Biomed Eng 2013;16(Suppl. 1):104-5.

[22] Borg G, Hassmen P, Lagerstrom M. Perceived exertion related to heart rate and blood lactate during arm and leg exercise. Eur J Appl Physiol Occup Physiol 1987;56(6):679-85.

[23] Jordan K, Challis JH, Newell KM. Long range correlations in the stride interval of running. Gait Posture 2006;24(1):120-5.

[24] Peng CK, Mietus J, Hausdorff JM, Havlin S, Stanley HE, Goldberger AL. Longrange anticorrelations and non-Gaussian behavior of the heartbeat. Phys Rev Lett 1993;70(9):1343-6.

[25] Bergstra SA, Kluitenberg B, Dekker R, Bredeweg SW, Postema K, Van den Heuvel ER, et al. Running with a minimalist shoe increases plantar pressure in the forefoot region of healthy female runners. J Sci Med Sport 2014.

[26] McCallion C, Donne B, Fleming N, Blanksby B. Acute differences in foot strike and spatiotemporal variables for shod, barefoot or minimalist male runners. J Sports Sci Med 2014;13(2):280-6. 